\title{
MAGNIFICATION AIDS IN ENDODONTICS: A REVIEW
}

\author{
Abdul Aziz Abdul Rahman AlEid*
}

\begin{abstract}
Objective: The purpose of this review for the magnification aids in endodontics was to describe in detail various magnifying devices, their use and the advantages and disadvantages in clinical surgical and conventional endodontic therapy with recent innovation and technological advancement in magnifying
\end{abstract}

Methodology: The author searched Qassim university library relevant journal articles manually and the website MEDLINE, Clinical key, EBSCO, google Scholar, Cochrane. The keywords for the search articles were dental operating microscope, dental magnifying loupes, dental endoscopes, dental orascope, root canal treatment, endodontic micro-surgeries magnification devices. The relevant articles describing the magnification devices were considered for the review.

Conclusion: The literature on the magnification devices is very limited for the most recent magnifying devices and their use is also very limited to the elite and developed countries in endodontic practice. However, dental operating microscope, being considered as standard of care, is commonly available but are less frequently used routinely. Dental magnifying loupes are with low magnification. However, its use is common due to low price and easy to use. Randomized clinical trials, comparing between these magnifying devices for precision and root canal treatment outcome is the need of the hour. Conventional Endodontic therapy and surgical endodontics should apply magnification devices to be used in clinical practice routinely.

\section{INTRODUCTION}

Magnification is the process of visualization of any structure or object in its enlarged, more detailed and in-depth the field seen by eyes. Naked eyes can see and have the resolution power of $0.2 \mathrm{~mm}$, that is when two lines or point are viewed and they are 0.2 $\mathrm{mm}$ apart normal eyes will most probably see them as one line or point, furthermore, if the distance between lines or points become $0.1 \mathrm{~mm}$ it will be difficult for most of the normal eyes to distinguish these minute closely placed entities. In routine dental practice microleakage and bacterial ingress, minute deleterious defect, an open margin of the restoration or for that matter the thickness of the luting cements of $25 \mu \mathrm{m}$ that will be about $0.025 \mathrm{~mm}$ that cannot

* Assistant Professor and Vice Dean academic affairs, Department of conservative dental science, College of Dentistry, Qassim University. 
be visualized by the normal resolving power of an unaided naked eye. Therefore, with an operating surgical microscope will be extremely beneficial aid for the eyes that can intensify and graciously increase the resolving power from $0.2 \mathrm{~mm}$ to $0.006 \mathrm{~mm}$ or $6 \mu \mathrm{m}$, a magnificent enhancing vision of the viewed structure entity. ${ }^{(1)}$ to Compound microscope invented by a physicist and biologist Robert Hooke, around three and half century back, he viewed a minute tissue structure that he called it a cell ${ }^{(2)}$. Need for magnification took its steps into dentistry with magnifying lens and latter with sophisticated newer magnifying gadgets. Endodontist had relied on the tactile sense, the radiographic image and the normal vision to develop a mental image of the root canal, straight or curved, without actually seeing deep down the canal. With the advent of magnifying equipment there is improvement in visualization of root canals and more better chances to locate minute canal orifice canal, intricate and highly complex internal anatomy of root canal morphology. Magnification will enhance the given object details with the help of an optical instrument depending on the power of magnification of that instrument. With the aim of high quality care treatment more innovative and high quality optical magnification equipments and devices are being more commonly used in dental practice for precision work $^{(3)}$. More accurate and precise operative procedures, illumination and magnification enhances diagnostic ability improvement, stable vision without movements, good working posture are few of the benefitting advantages for the use of magnification and good illumination. In endodontics micro marginal gap, microfractures, isthmus, ledge, perforations and instrument separation are few examples were magnification will be effective in successful outcome. With vision enhanced with the magnification aid is well known and are recorded in medical and as well as dental sciences ${ }^{(4,5)}$

Pioneering endodontists and other practioners used magnifying loupes, with lower magnification resolution power till 1980, than the surgical operating microscope with higher magnification resolving power was introduced to endodontic practice $^{(6)}$

The optical principles that are important factors in improvement of visual visualization are stereposis, range of magnification, depth of field, resolving power working distance, spherical and chromatic aberrations, ergonomics, eyestrain, hed, neck fatigue and the cost.

Individual variations are a common findings for the natural vision acuity and it will further decreased as the age advances over time ${ }^{(7,8)}$

With age constrained accommodation as well as reduced contrast sensitivity is usually accompanied with presbyopia meaning, natural part of aging with loss of near focusing ability near sightedness, farsightedness and astigmatism ${ }^{(9)}$ In the field of dentistry the first coined microdentistry and dental operating microscope along with the accessory accompanying equipments was introduced in 1981 by Harvey Apotheker ${ }^{(10)}$ in its crude form that was poorly designed and was cumbersome and was clinically difficult to use in practice.Later in 1992 Dr. Gary Carr designed a more sophisticated operating microscope that is user friendly along with better illumination and greater magnification resolving power, that is easier to use in clinical practice. Therefore, fast acceptance of Dental operating microscopes not only in endodontics but literally all of the dental disciplines ${ }^{(11)}$

In a simulated clinical setting for the visual test in the root canal, intra-radicular, to study and understand the dental operating microscope and to establish its significance for the endodontic acuity. The test concluded that the visual acuity did not depended on the age of the operator or the locations within the root canal system when using Dental operating microscope, with naked eyes and with the use of Galilean loupes integrated with the light source were deficient could not aid in magnification 
of any measureable acuity in the root canal however young operators with less than 40 years of age were able to detect $0.06 \mathrm{~mm}$ orifice of the root canal with these loups. (Perrin et al. Vision and endodontics)

Endodontic visualization system

1. Dental loups/telescopes:

i) A diopter single flat plane lens.

ii) Surgical telescope two lens Galilean configuration system

iii) Surgical telescope Keplerian system configuration with prism roof design ${ }^{(12)}$

2. Dental operating microscope

3. Endoscope.

4. Oroscope.

Dental loups/telescopes: optical method of magnification will classify the magnifying loups into

---A Diopter single flat plane lens loups (i

Optical method of magnification is diopter, which is focusing distance between eyes and the object. 1dipopter is $100 \mathrm{~cm}, 2$ diopter is $50 \mathrm{~cm}$ likewise 5 diopter is $20 \mathrm{~cm}$. they are with minimal cost but are plastic and optically not very correct. For magnification of the entity the operate need to reduce the distance between the eyes and object to be viewed such a posture for musculoskeletal parts will cause stresses and postural complications hence they are less popular in clinical dental practices ${ }^{(13)}$

ii) Surgical telescope two lens Galilean configuration system ---

iii) Surgical telescope Keplerian configuration system with prism roof design.

Magnifying Loupes are more popular and more commonly used in endodontic surgeries. They are less expensive, smaller simple unit for magnification and provide good illumination. To overcome the disadvantage of diopter, occasioned by coming closer to the object, the loupes were developed in order to reduce the problem of proximity, musculoskeletal complications, eyestrain and also to decrease the field depth, that is to adjust the focusing lens system, without changing the position of the lens, on the objects which are either near or far away. As the field of view becomes small the depth of field become shallow or deceased. The depth of field will be about $12.5 \mathrm{~cm}$ for a $\mathrm{x} 2$ magnification loupe and likewise $6 \mathrm{~cm}$ for $\mathrm{x} 3.5$ loupe and $2.5 \mathrm{~cm}$ for $\mathrm{x} 4.5$ loupe. The distance between the operator and the object when using either Galilean configuration or Keplerian configuration (Figure 1 and Figure 2) is normally 28 to $38 \mathrm{~cm}^{(11)}$.

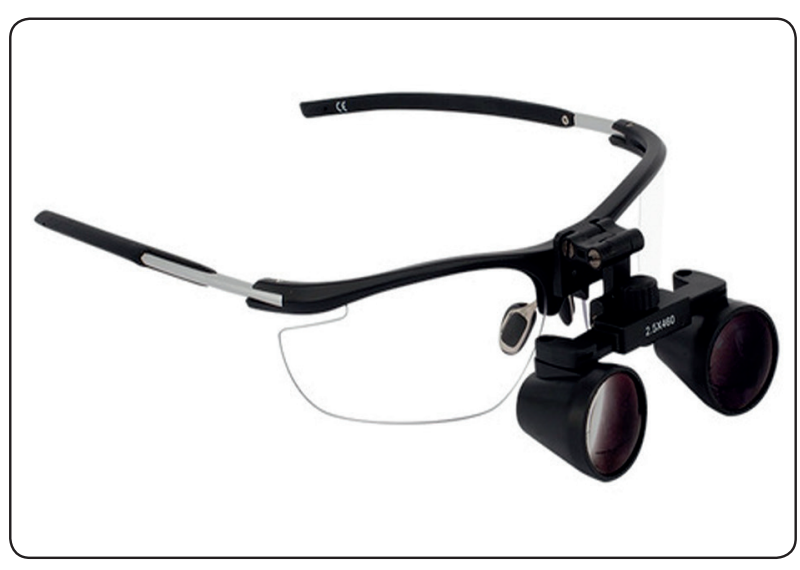

Fig. (1) Galilean flip-up loupes

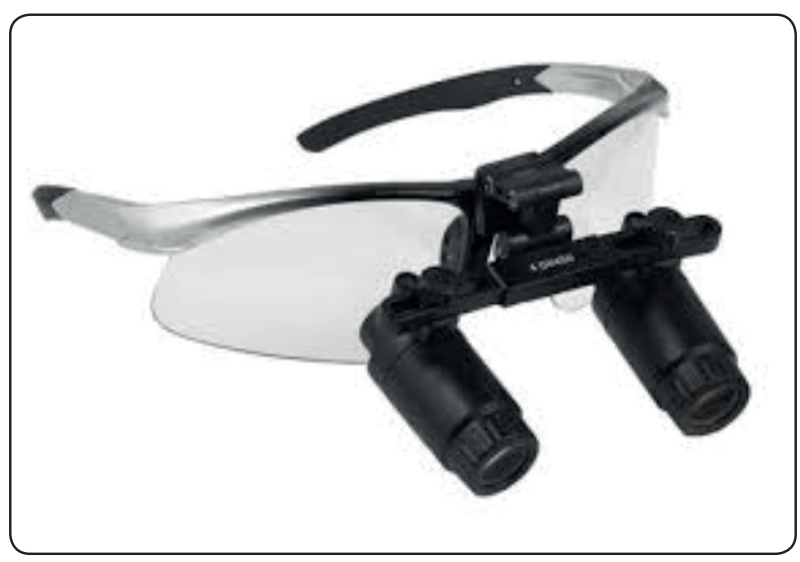

Fig. (2) Keplerian prims loupes

Loupes are made to view the object such that the eyes will converge to see through the lens angled inward (convergent optics) with two monocular 
Magnifying lens placed side by side. Whereas the compound loupes are with multiple lenses filled with air spaces between them but the more advanced magnifying optical loupes are the prism loupes. These prism magnification loupes are with refractive prism that can provide superior magnification. loupes have better field of depth, gives superior magnification up to $\mathrm{x} 4.5$, can be customized for individual requirement. However, greater than $\mathrm{x} 4.5$ higher magnification loupes will have limited field of view as well as depth of field, these are heavy, and can induce eye strain, head and neck as well as back strain with improper operator posture and use of these relatively heavier magnifying loupes ${ }^{(14)}$

Illumination plays an important role in visual acuity apparent resolution, where the two entities placed very near to each other are clearly visible as two distinct and as two separate entities. Inverse square law governs the light intensity, that is, if the distance between the source of light and object is decreased by one half the amount of light will increase four times at the object. For good illumination the loupes are fitted with operator's headlamps, which has short working distance of 13 to $35 \mathrm{~cm}$, and is placed in the center and can move with the operator's head movements accordingly and the fiberoptic cable that will transmits light and reduces heat levels ${ }^{(1)}$

Loupe (Figure 3) are similar to spectacles with added telescopic lens, not expensive, no special training required, wear and work, handling, carrying and maintenance is easy, can be stored in a small cupboard cabinet shelf. However, loupes have limited magnification of $\mathrm{x} 6$ and for higher magnification requires heavier loupes and not practical, it also have limited intensity head-lamps for illumination.

\section{Dental Operating microscope:}

Dental operating microscope was first developed by Apotheker in early $1981^{(10)}$ in a crude form,

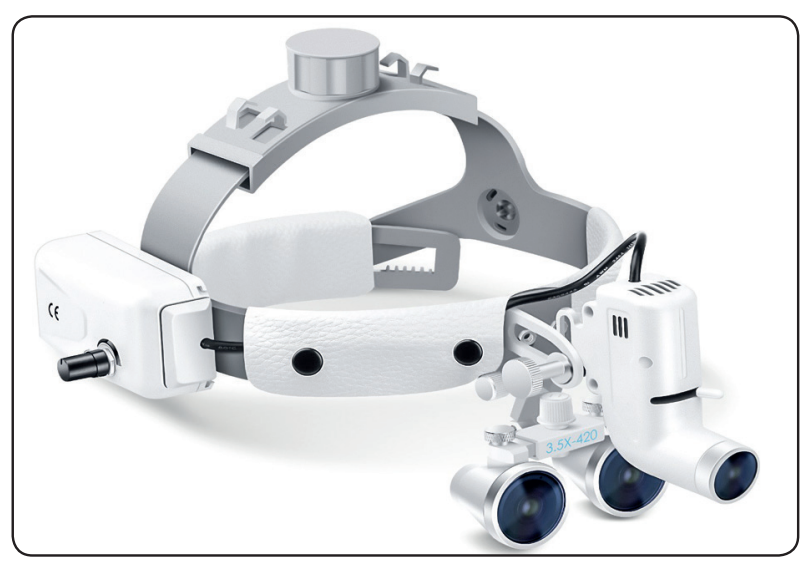

Fig. (3) Surgical binocular with headband loupes and LED light

cumbersome to use and not practical in using for routine dental practice. A decade later an electronics technician in the US navy, dentist and a renowned endodontist and a great innovator of dental operating microscope Dr. Gary Carr gave the microscope an improved ergonomic with better configuration and was more an user friendly practically easier to use in routine endodontic clinical procedures. Due to its advantages of magnification and routine use, not only endodontics but other branches of dentistry are inclined to introduce operating microscope for clinical practice (figure 4 ).

The dental operating microscope are either wall mounted, ceiling mounted or movable floor models. It has three main parts, the supportingstructure, body of the microscope includes eyepiece lenses and prism, magnification changer, objective lens, , illumination and accessories. For using dental microscope efficiently in routine endodontic practice its regular use and advanced training is required. The magnification for most of the endodontic treatment procedures can be done under x 10 to $\mathrm{x} 15$. However, for greater details and some of the procedures may require the magnification as high as $\mathrm{x} 30$.

Eyepiece is the part of the microscope and its power will determine the magnification which is available $\times 6 \times 10 \times 12.5 \times 16 \times 20$ and the Diopter 


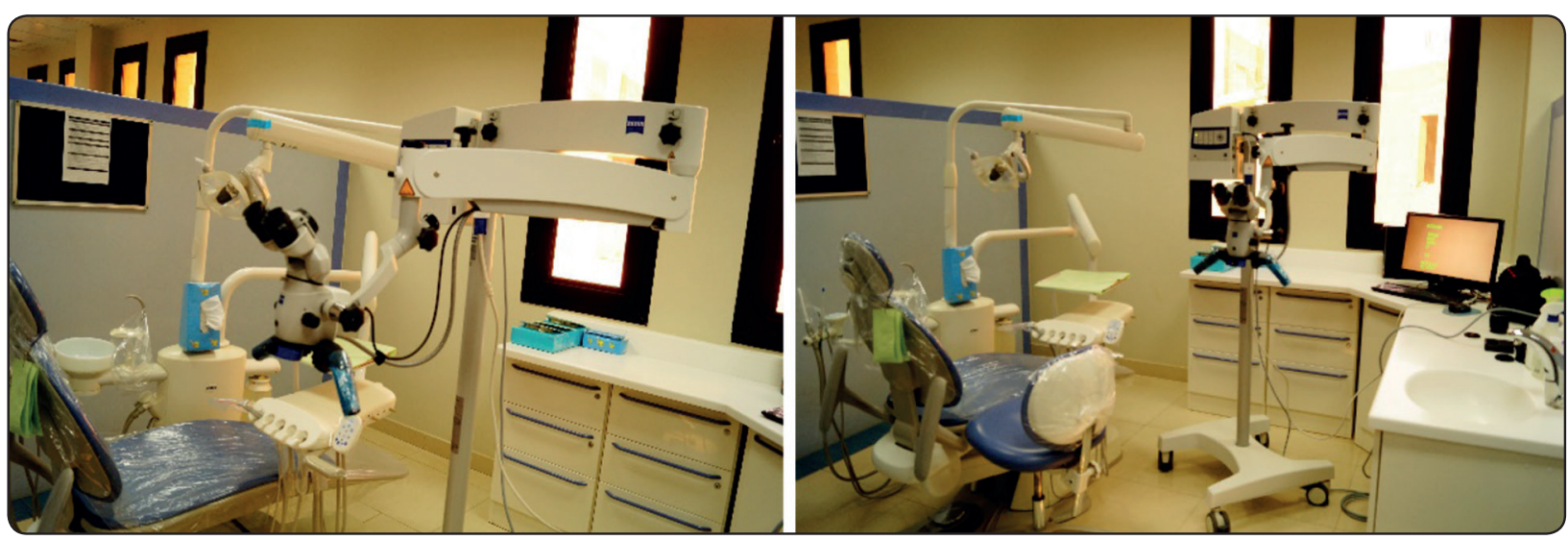

Fig. (4) Dental Surgical microscope

range is -5 to +5 for adjustment ofeye-lenses. The binocular holds the Eyepiece and it allows interpupillary distance adjustments are often supplied in different focal length. The focal length required for focusing on the work area between the lens and the operating field which is 125 to $160 \mathrm{~mm}$ the focal length can be adjusted manually o with small knob until the 2 divergent circles of light combines to form a single clear focus area. Binoculars are available as inclinable, inclined or straight tubes that hold the eyepieces. The more preferred for dental operating microscope is inclinable tubes due to its adjustment that is most comfortable and suitable for the operator.

The magnification changers positioned on the sides of microscope head either it can be adjusted manual available with 3,5 or 6 steps or of power zoom changer.

The objective lens providea range of 100 to 400 $\mathrm{mm}$ of focal length, but for most of the endodontic procedures $200 \mathrm{~mm}$ of focal length is adequate that will allow $20 \mathrm{~cm}$ working distance between the DOM and surgical field (Figure 5).

Four factors combination will determine the total magnification--TM of anoperating microscope. These are

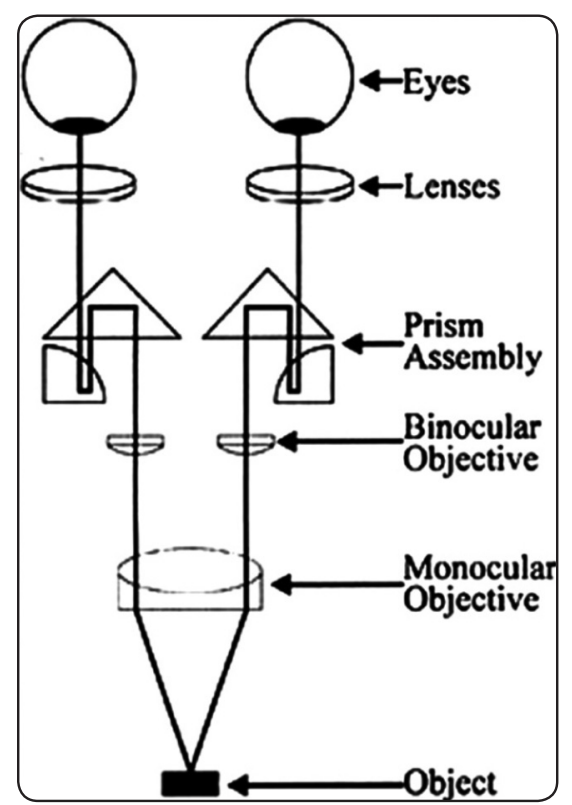

Fig. (5) Galilean optics. Parallel optics enables the observer to focus at infinity, relieving eyestrain. Schematic presentation Body of DOM(1).

NOTE: Image is taken from the article by: Carr GB, Murgel CA: The use of the operating microscope in endodontics. Dent Clin North Am 2010;54:191-214

i) Focal length of the binocular-FLB. ii) focal length of the objective lens-FLOL. iii)Eyepiece power-EP. iv) magnification changer factor-MF. Thus the formula for the total magnification can be equated as follows: $\mathrm{TM}=(\mathrm{FLB} / \mathrm{FLOL}) \mathrm{X} \mathrm{EP} \mathrm{X}$ $\mathrm{MF}^{(14)}$ 
Illumination: an important factor :the source of light confocal illumination module which is coaxial in such a way that the path of the light and the optical path is same as the visual path, as both the paths are confocal that eliminate an shadow and it provides superior illumination than the angulated light path from the earlier versions of microscopes.

Accessories: In order to deflect a certain percentage of the light from the eyepiece towards the accessories, a beam splitter can be placed between the binoculars.

\section{Accessories:}

Beam splitter: is placed between the binocular and the magnification changer which provide light for the eyepieces and accessories by deflection $50 \%$ that is half of the light in 50:50 ratio. A camera or a video camera can be attached to the beam splitter for recording the procedure and for documentation.

The law of ergonomics in dental operating microscope:

As explained by Dr. Gray for skillful use of dental operating microscope it should be used for complete procedure from the beginning to the finished end by using the microscope regularly in routine endodontic procedure will provide refinement of ergonomics and also improve visual skills to a greater level. To understand and know efficient workfield for using a dental operating microscope it is essential to gain basic knowledge of ergonomics motions:

Class I motion: movement that involves only fingers for working

Class II motion: movement that involves fingers and wrists only

Class III motion: movement that involves movements involving and originating from elbow.

Class IV motion: movement that originates from the shoulder for working

Class V motion: movement that involves waist either bending and/or twisting.
By using dental operating microscope it will provide improvement in vision with more accurate diagnosis that will help to achieve better treatment result outcomes and can be comfortable ergonomic posture.

when dental operating microscope is used it must be adjusted and maneuvered as follows:

Step1: Proper operator position

Step2: Positioning of the patient in proper working range under microscope

Step3: Positioning of dental operating microscope and focusing on the targeted surgical field

Step4: Adjusting the eyepieces for interpupillary distance

Step5: Fine positioning of the patients under microscope

Step6: Parfocal adjustment and finally

Step7: Fine focus adjustment of the surgical field

Step8: In case of four handed dentistry assistant side microscope is adjusted.

Uses and Advantages of Dental operating microscope:

Magnification with dental operating microscope can be utilized in routine endodontic treatment procedures to identify and detect canal orifice like MB2 in maxillary molars and /or $\mathrm{C}$ shaped canals in mandibular molars etc., to detect perforations in the floor or root-canals, to detect hard- to-find calcified canals, provide clear vision for retrieval of post and/or fracture-separated-broken endodontic instruments in the root canals and to provide precision to the high quality root canal therapy. For minimal invasive apical surgeries, to detect and identify the root tip/ apex, resection at a $180^{\circ}$ or less of involved root apex, even the isthmus can the detected and allows precision in retrograde filling 


\section{Orascope and Dental Endoscope}

The use of orascope (Figure 6 and Figure 7) for endodontic treatment either conventional endodontics or for surgical endodontic procedures is called as orascopy. ${ }^{(16)}$ An orascope, used for the intra-canal visualization, has a tip diameter of 0.8 $\mathrm{mm}$, length of $15 \mathrm{~mm}$ and a zero degree lens and for light source for illumination is provided by the light transmitting fibers that are places around the visual fibers. The diameter of these visual and light transmitting fibers range from 3.7 to $5 \mu$ diameter and consists of about 10,000 parallelly placed fibers. The three components of orascope and endoscope, that work in conjunction and synchrony, are a camera, light source and a monitor, but a printer and / or a digital recorder can installed for documentation ${ }^{(17,18)}$

Innovation and technology is advancing rapidly and so is in the field of visualization a more versatile recent addition is an compact fiber optic endoscope for use in endodontic procedures ${ }^{(19,20)}$.

Endoscope (Figure 8 and Figure 9) is with one great advantage over the dental operating microscope, enables the operator to view the surgical area field different various angles and distances not losing any field depth as well as the focus. Unlike the dental operating microscope, endoscope is peculiar

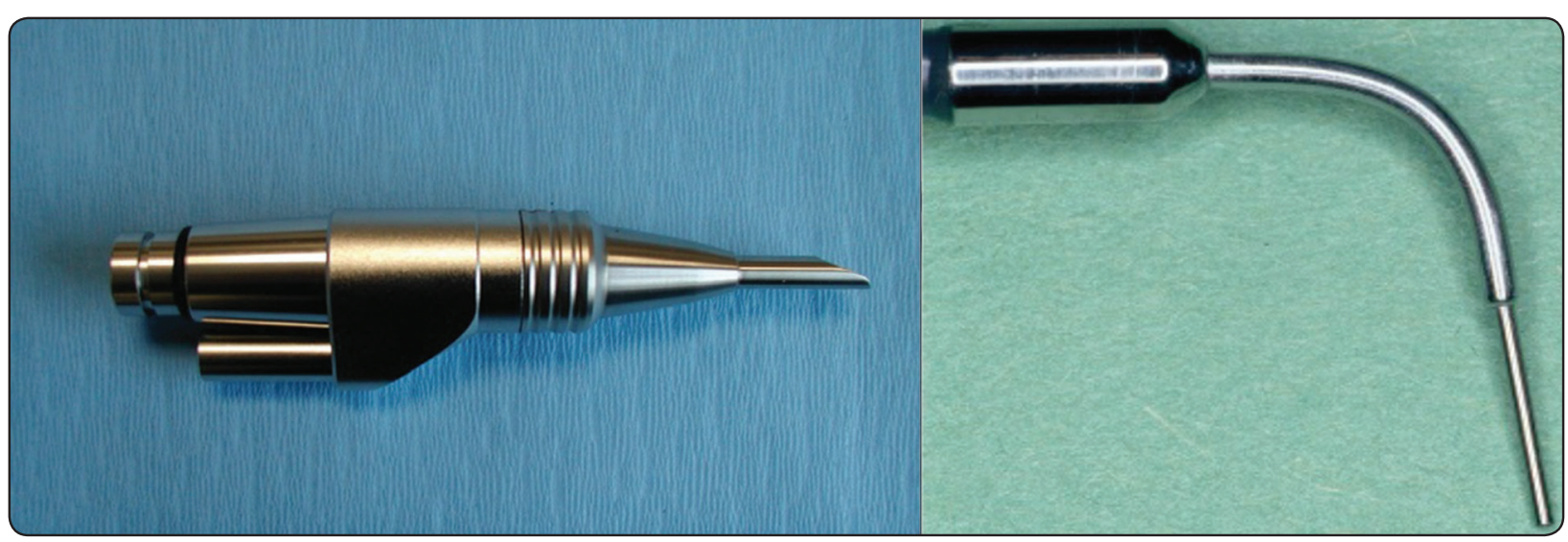

Figure $6 \& 7$ : A rod-lens endoscope (JEDMED, St. Louis, MO) and A Orascope.

NOTE : Images are taken from the article by: Bahcall JK. ${ }^{(15)}$ Visualization in endodontics. Eur J Gen Dent. 2013;2:96-101.

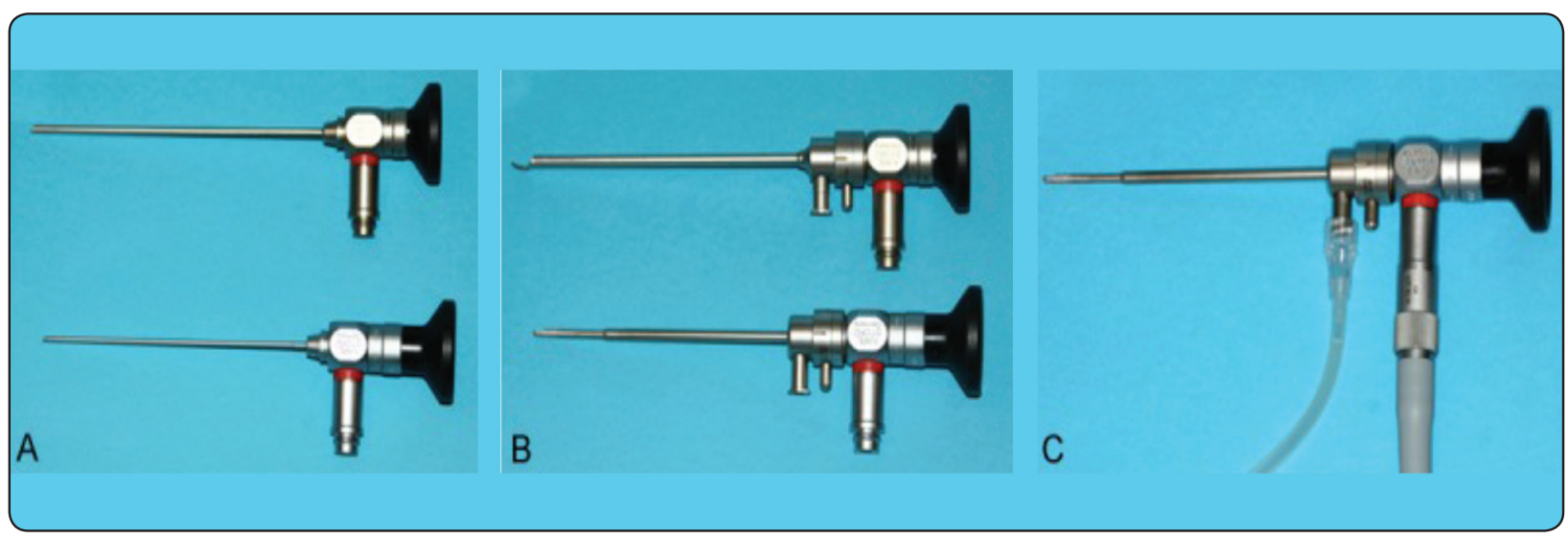

Fig. (8) Rigid endoscopic systems: A. Optics (Rigid) $2.7 \mathrm{~mm}$ and $1.9 \mathrm{~mm} .30$ degree view angle. B. Optic assembled with its support. C. Connection to the light source and irrigation. ${ }^{(24)}$.

NOTE: Image are taken from the article by: Wilfried Engelke, Carolina Leiva, Gunar Wagner Víctor Beltrán.: In vitro visualization of human endodontic structures using different endoscope systems. Int J Clin Exp Med. s2015; 8(3): 3234-3240 


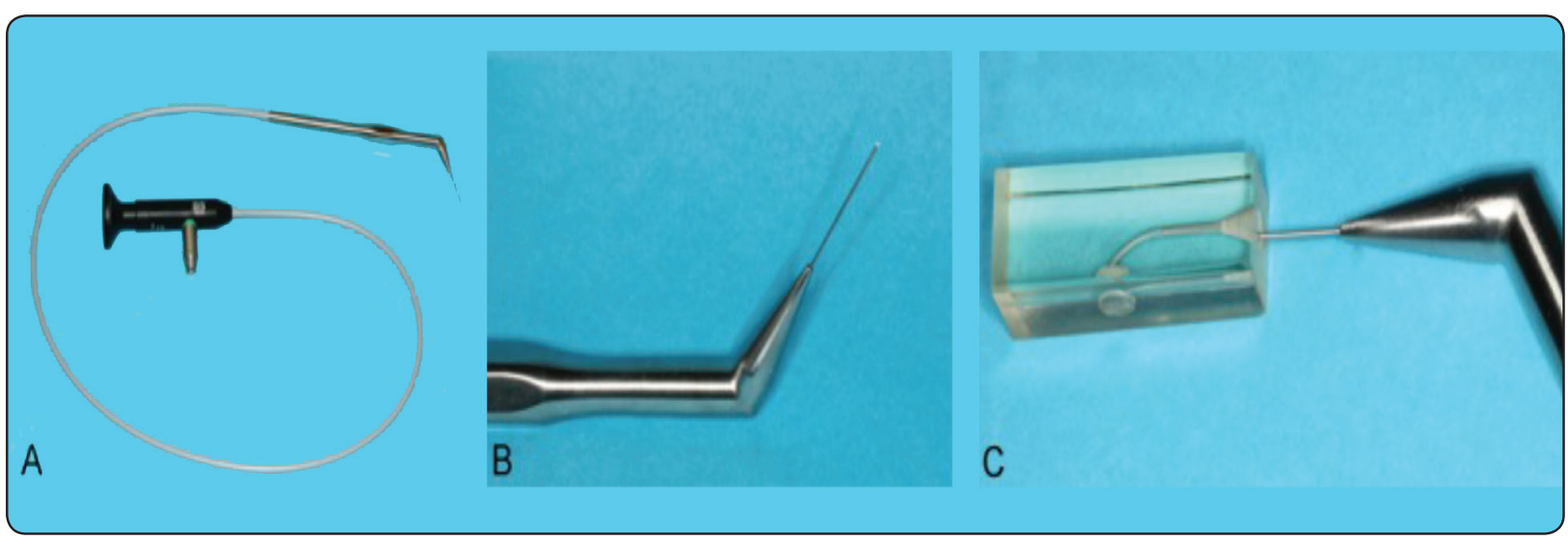

Fig. (9) Semiflexible Endoscope: A. Semiflexible Endoscope, outer diameter $0.5 \mathrm{~mm}$ and 0 degree views angle. B. Angled handpiece with incorporated fibre optic and light transmission. C.Endoscope inserted into the root canal of a phantom. ${ }^{(24)}$

NOTE: Image are taken from the article by: Wilfried Engelke, Carolina Leiva, Gunar Wagner Víctor Beltrán.: In vitro visualization of human endodontic structures using different endoscope systems. Int J Clin Exp Med. 2015; 8(3): 3234-3240

for non-fixed field vision ${ }^{(21)}$. An added advantage for endoscope is that it is comfortable for use and working with it is more faster and can be adjusted with one finger for zooming as well as focusing. However, for dental operating microscope to focus or zoom single or both hands are needed and requires to stop while working to adjust manually, whereas for endoscope, operator can hold with pen grasp the endoscope and camera and adjust focus and zoom with a finger. Also when endoscope is moved out of the operating site it can be very easily readjusted to surgical operating site. Endoscopes are indeed versatile making it possible to see and view behind the root where periapical lesions surrounds the root tips that can be treated, but with the use of dental operating microscope it will be rather difficult for operator to visualize with small retro mirror. Also allows visualization in presence of irrigation with sterile water that kept the lens clean and prevents overheating when retro tips are used for apical root preparation, unlike the retro mirrors that will require frequent cleaning of these mirrors when used with dental operating microscope $\mathrm{e}^{(22)}$

Endoscope for surgical endodontics, recommended by the pioneer operators, is with 4.0 $\mathrm{mm}$ lens, with angulation of $30^{\circ}$ and length of 6 $\mathrm{cm}$. in case if the angulations was increased more than 30 degree the distortion of image had fish eye appearance. However, with improvements in design with innovative technology smaller endoscope with shorter length and increased angulation for lens more than 30 degree is possible without any fish eye image effect ${ }^{(23)}$ Rod-lens endoscope with $2.7 \mathrm{~mm}$ diameter tip, with $70^{\circ}$ angulation and $3 \mathrm{~cm}$ length are available.

\section{CONCLUSION}

The literature on the magnification devices is very limited for the most recent magnifying devices and their use is also very limited to the elite and developed countries in endodontic practice. However, dental operating microscope, being considered as standard of care, is commonly available but are less frequently used routinely. Dental magnifying loupes are with low magnification. However, its use is common due to low price and easy to use. Randomized clinical trials, comparing between these magnifying devices for precision and root canal treatment outcome is the need of the hour. Conventional Endodontic therapy and surgical endodontics should apply magnification devices to be used in clinical practice routinely. 


\section{REFERENCES}

1. Carr GB, Murgel CA: The use of the operating microscope in endodontics. Dent Clin North Am 2010;54:191-214

2. Ham AW: Histology 7th Ed. Philadelphia; PA: Lippincott,Willaim and Wilkins, 1974.

3. Bahcall J, Di Fiore P, Poulakidas T.: An endoscopic technique for endodonticsurgery. J Endod 1999: 25: 132-135.

4. Castellucci A: Magnification in endodontics: the use of the operating microscope.PractProcedAesthet Dent 2003; 15:377-84.

5. Kim S, Baek S.: The microscope and endodontics. Dent Clin North Am 2004; 48:11-8.

6. Koch K. : The microscope: its effect on your practice. Dent Clin North Am 1997;41: 619-26.

7. Burton JF, Bridgman GF : Presbyopia and the dentist: the effect of age on clinical vision. International Dental Journal $1990: 40,303-12)$

8. Eichenberger M, Perrin P, Neuhaus KW, Bringolf U, Lussi A : Influence of loupes and age on the near visualacuity of practicing dentists. Journal of Biomedical Optics,2011 :16, 035003).

9. Sia DI, Martin S, Wittert G, Casson RJ : Age-relatedchange in contrast sensitivity among Australian maleadults: Florey Adult Male Ageing Study. Acta Ophthalmologica 2012: 91, 312-7.

10. Apotheker H, Jako GJ. A microscope for use in dentistry. Journal of Microsurgery. 1981;3:7-10

11. Carr GB. : Microscopes in endodontics. Journal of California Dental Association. 1992;20:55-61.

12. Carr GB. : Magnification and illumination in endodontics. In: Hardin FJ, editor. Clark's clinical dentistry, vol. 4. St Louis, MO: Mosby; 1998.p. 1-14\}.

13. Weller N, Niemczyk S, Kim S.: The incidence and position of the canal isthmus: part 1. The mesiobuccal root of the maxillary first molar. J Endod 1995;21(7): 380-3.

14. Castellucci A. Magnification in endodontics: the use of the operating microscope. Practical Procedures and Aesthetic Dentistry. 2003;15:377-84).

15. Bahcall JK: Visualization in endodontics. Eur J Gen Dent; 2: 96-101.

16. Bahcall J and Barss J 1999. Orascope: Vision for the millennium. Part II. Dentistry Today 18, 82-85.

17. Bahcall JK, Barss JT. Orascopic endodontics: changing the way we "think" about endodontics in the 21 st century. Dent Today 2000; 19(5): 50-55.

18. Bahcall JK, Barss JT. Endodontic therapy using orascopic visualization. An update. Dent Today2003; 22(11): 95-98.

19. TaschieriS, Del FabbroM, Testori T,FrancettiL, Weinstein RL. Endodontic surgery using two different magnification devices. Preliminary results of arandomized controlled study. J Oral MaxillofacSurg 2006: 64: 235-242

20. (von ArxT, Walker WA. Microsurgical instruments for root-end cavity preparation following apicoectomy: a literature review. Endod Dent Traumatol 2000: 16: 47-62.

21. BahcallJ, Di Fiore P, PoulakidasT. An endoscopic technique for endodontic surgery. J Endod 1999: 25: 132-135.

22. Taschieri S, Del Fabbro M, Testori T, Weinstein R. Microscope versus endoscope in root-end management: a randomized controlled study. Int J Oral Maxillofac Surg 2008;37:1022-6

23. von Arx T, Hunenbart S, Buser D. Endoscope- andm video-assisted endodontic surgery. Quintessence Int 2002; 33(4): 255-259.

24. Wilfried Engelke, Carolina Leiva, Gunar Wagner Víctor Beltrán.: In vitro visualization of human endodontic structures using different endoscope systems. Int J Clin Exp Med. 2015; 8(3): 3234-3240 